\title{
Transport Properties of Rippled Graphene
}

\author{
M. ZWIERZYCKI*
}

Institute of Molecular Physics, Polish Academy of Sciences, M. Smoluchowskiego 17, 60-179 Poznań, Poland

\begin{abstract}
It is common to describe graphene as ideally flat plane, however there exists both theoretical and experimental evidence that it is most usual to find it in a rippled state. The ripples can be either induced by the substrate or formed spontaneously in suspended graphene. The lateral size of such features ranges between several and tens of nanometers with the height of up to $1 \mathrm{~nm}$. It has been suggested that the presence of ripples could be one of the factors ultimately limiting mobility of carriers and that it may be also responsible, by introducing an effective gauge field, for the lack of weak localization observed in certain graphene samples. In the present contribution the transport properties of the rippled graphene are studied theoretically starting with the simple case of one-dimensional modulation. Using either single-band or the full $s p^{3}$ tight-binding Hamiltonians we compare and discuss the importance of two ripple-related mechanisms of scattering: the variation of interatomic distances and hybridization between $\pi$ and $\sigma$ bands of graphene.
\end{abstract}

PACS: 81.05.ue, 72.10.Fk, 72.80.Vp

\section{Introduction}

The discovery of graphene, two-dimensional (2D) carbon allotrope, in 2004 [1] arose high hopes for its application in future electronic devices. The reason for this is high (exceeding $10^{5} \mathrm{~cm}^{2} /(\mathrm{V} \mathrm{s})$ ) mobility of carriers whose density and character (electrons or holes) can be controlled either by electric or chemical doping. The remarkable transport properties of graphene are related to peculiarity of its electronic structure, namely the fact that low-energy excitations can be described as massless, chiral Dirac fermions albeit with the speed of light replaced by 300 times lower Fermi velocity [2]. This leads to a range of interesting phenomena like finite minimal conductivity, the Klein paradox or the anomalous quantum Hall effect which can be observed even at room temperatures (see [3] for recent review).

It is common to assume in the theoretical studies of graphene that it forms a perfectly flat plane. There exists however a convincing evidence that it is not so. Spontaneous rippling has been seen both in experiment [4] and simulations [5] for flakes of suspended graphene. In the more usual case of graphene on a substrate, the structural corrugations of the latter are followed, at least partially, by the graphene sheet [6]. In some cases both substrate induced and intrinsic modulations can be observed [7]. In general the ripples appear to be a common feature of graphene samples. Lateral sizes of modulations can range between several and tens of nanometers and the height can be of up to $1 \mathrm{~nm}$.

\section{Theory}

Three different mechanisms can modify electronic structure of thus deformed graphene: (i) alteration of interatomic distances leading to the change of the hopping integrals, (ii) hybridization between $\sigma$ and $\pi$ bands

* e-mail: maciej.zwierzycki@ifmpan.poznan.pl which are strictly orthogonal in planar graphene and (iii) local shifts of the Fermi level (neutrality point) leading to the formation of the electron and hole pools [8]. Interestingly, in the effective mass approximation, the first two effects can be described in terms of an inhomogeneous gauge field [9]. The presence of such pseudomagnetic field could lead to the formation of the flat Landau levels (LL) whose presence was predicted theoretically using either simple tight-binding model [10] or ab initio calculations [11].

It has been suggested that rippling can be responsible, by introducing intrinsic pseudomagnetic field, for the absence of the weak localization in some samples [12] and also that it can be an ultimate factor limiting mobility of carriers in graphene [13].

The aim of this paper is to study the transport properties of graphene in the presence of a simple sinusoidal modulation along the transport direction. In the following paragraphs we will discuss the theoretical methods employed and then present the results of calculations for a system consisting of either single or multiple periods of such modulation.

\section{Calculations}

The calculations were performed using tight-binding Hamiltonian in the Slater-Koster parameterization as given in Ref. [14] (non-orthogonal basis set, nearest neighbours hopping (n.n.) only). Two specific models were considered: $s p^{3}$ with both $\pi$ ( $p_{z}$ orbitals) and $\sigma$ $\left(s, p_{x}, p_{y}\right)$ bands present ( $x y$ being the graphene plane) and single-band model restrained to the $\pi$ band. In the case of the latter all hybridization effects are disregarded and the only effect of the deformation of the graphene plane is the change of interatomic distances leading to the modification of the hopping and overlap integrals. A general $t_{l, l^{\prime}}(r) \sim r^{-l-l^{\prime}-1}$ scaling rule is used to model this effect. By using two models it is therefore possible to distinguish between the effects of $\sigma-\pi$ hybridization, 
included naturally by using the Slater-Koster form of the Hamiltonian, and the scaling of the hopping constant, i.e. two of the effects described above. The presence of the ripples does not change, energy-wise, the position of the neutrality point of graphene when only n.n. hopping is included, hence the third effect is not present. Let us note that the non-orthogonality of the basis set leads to explicit breaking of the electron-hole symmetry.

A simple one-dimensional ripple, $h(x)=$ $h_{0} \sin (2 \pi x / L)$, is considered with $h$ being the deviation from the $x y$ plane and $x$ the direction of the transport. The calculations were performed for two periods, $L$, of $60 a_{0}$ and $120 a_{0}$, where $a_{0}=1.42 \AA$ is the n.n. distance for flat graphene. The graphene plane is oriented so that the "armchair" direction coincides with that of the ripple $(x)$. Translational invariance is preserved in the lateral direction (along $y$ ), therefore $k_{y}$ remains a good quantum number.

The transport properties were studied for a system consisting of a single period of modulation (scattering region) sandwiched between flat graphene electrodes. The effect of doping was simulated, independently for electrodes and scattering region, by modification of the on-site elements of the Hamiltonian. The local chemical potential, $\mu$, defined with respect to the touching point of the valence and conduction bands (i.e. neutrality point), was fixed to $\mu=1 \mathrm{eV}$ in the electrodes. The LandauerBüttiker conductance $(G)$ and Fano factor $(F)$ were then calculated using the following formulae:

$$
G=\frac{e^{2}}{2 \pi h} \int \mathrm{d} k_{y} T\left(k_{y}\right) ; \quad F=\frac{\int \mathrm{d} k_{y} T\left(k_{y}\right)\left[1-T\left(k_{y}\right)\right]}{\int \mathrm{d} k_{y} T\left(k_{y}\right)}
$$

as function of the $\mu$ in the central region. The $k_{y^{-}}$ -dependent transmission probability, $T\left(k_{y}\right)$, was determined using a wave function matching method described in [15] and similar conceptually to the approach adopted in $[16,17]$. The reported values of the conductance are per length of the two-dimensional unit cell in the direction perpendicular to the transport equal to $\sqrt{3} a_{0}$.

\section{Results}

The results of band structure calculations for a structure with period of $L=60 a_{0}$ are shown in Fig. 1 (along $y$ direction and with $\left.k_{x}=0\right)$ for vertical amplitudes $\left(h_{0}\right)$ of 4,12 , and $20 \AA$, respectively. Initially, for small modulation (top part), the effect of rippling is to shift the Dirac cones away from its original position, shown in the plots by a vertical line. The first indications of flattening of a central bands are seen for $h_{0}=12 \AA$ (middle part) and for $h_{0}=20 \AA$ we do see a fully developed flat band, that is $n=0$ Landau level in pseudomagnetic field interpretation. Additionally, as the modulation increases the states in the vicinity of the neutrality point become increasingly localized in the $x$ direction which leads to the energy gaps opening between sub-bands visible in Fig. 1. The results shown using solid lines correspond to single-band model. In the middle panel $s p^{3}$ results are also

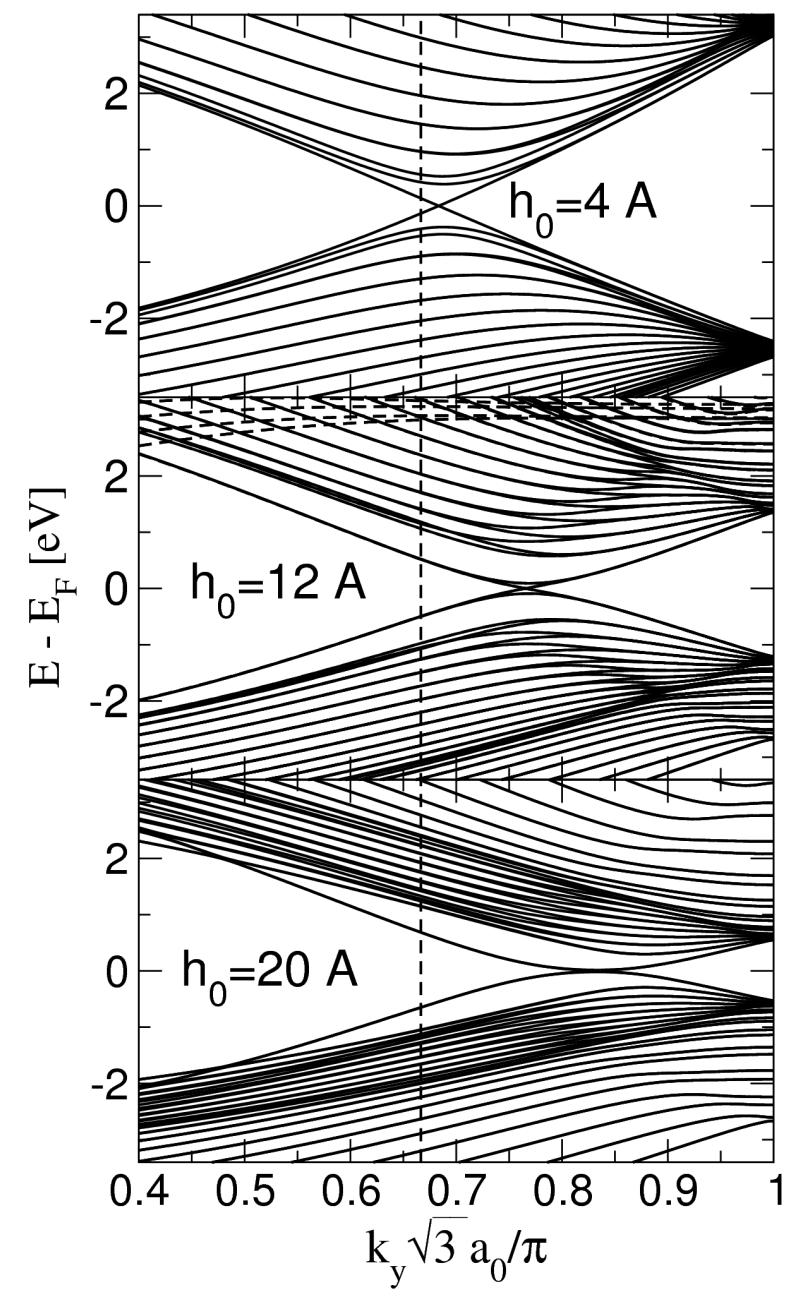

Fig. 1. Band structures of graphene with a one-dimensional sinusoidal ripple. The period of modulation is equal to $L=60 a_{0}$ and the vertical amplitudes are indicated in the plots. In the middle part the results of $s p^{3}$ model are plotted using dashed lines. The vertical line indicates the position of the original Dirac cone for a flat structure.

shown using dashed lines. There are no visible differences for the energies of $\pi$ bands (the lines overlap). The $\sigma$ bands however are brought much closer to the Fermi level than in the flat graphene and are now visible in the upper part of the plot. Similar results were obtained for $L=120 a_{0}$ ripples (not shown), however the formation of LL state occurs for stronger modulations $\left(h_{0}=20 \AA\right.$ and above). The results presented here show a good overall agreement with $a b$ initio calculations of Ref. [11].

The calculated conductances are shown in Fig. 2. Concentrating first on the top part $\left(L=60 a_{0}\right)$ we observe an interesting evolution of the conductance curves with the increasing amplitude of the modulation. The curve for flat graphene $\left(h_{0}=0 \AA\right)$ follow, for the most part, the Sharvin conductance (proportional to the number of propagating modes) of the central part. The exception is the area around neutrality point $(\mu=0 \mathrm{eV})$ where 


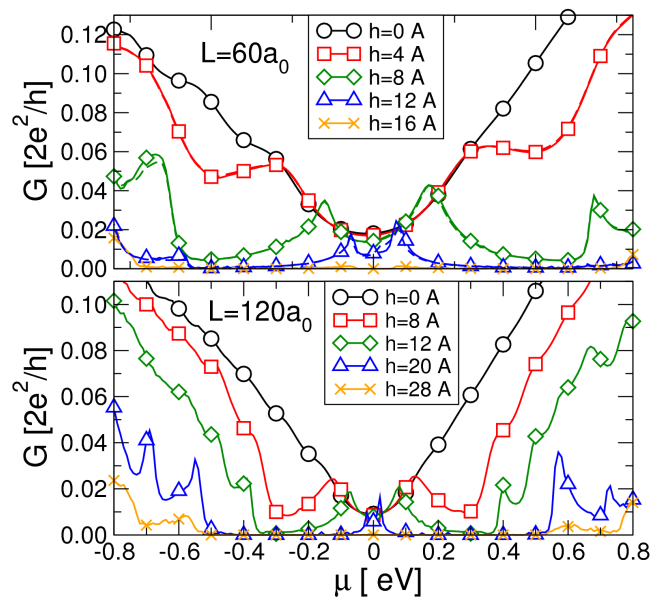

Fig. 2. The conductance through a single period of modulation for $L=60 \AA$ (top part) and $120 \AA$ (bottom part) as a function of the chemical potential in the central region.

the strong contribution from the evanescent states leads to the rounding of the curve. As the vertical amplitude $\left(h_{0}\right)$ increases, the conductance in this region is initially unchanged and starts decreasing meaningfully only for amplitudes over $8 \AA$. At the same time the conductance away from neutrality point undergoes dramatic changes with two satellite regions of lowered conductance developing around $\mu= \pm 0.5 \mathrm{eV}$. These are getting lower and broader with increasing $h_{0}$. At $h_{0}=12 \AA$ only the central region around neutrality point remains different from zero and even that is gone, for the range of energies studied, by the time we reach $16 \AA$. The results for single-band (solid line) and $s p^{3}$ models (dashed line) are both shown but the curves are almost impossible to distinguish on the scale of the plot. This indicates that the transport properties are defined mostly by the effect of varying bond lengths while $\sigma-\pi$ hybridization plays only minor role. The results of $L=120 a_{0}$, shown in the bottom part, are qualitatively very similar. The structure of the conductance curves can be understood in terms of the underlying electronic structure (Fig. 1). The low conductance regions around neutrality point correspond to the energy gaps opened by the modulation and the peaks indicate the sub-bands crossing the Fermi level.

The Fano factors shown in Fig. 3 for $L=60 \AA$ indicate a sub-Poissonian $(F<1)$ character of a shot-noise. The results for region around $\mu=0$ remain remarkably independent of the modulation amplitude. In particular we observe practically constant maximum value of $1 / 3$, in agreement with flat-graphene results of [17]. Outside of the central region $F$ assumes initially, for small $h_{0}$, lower values also in line width [17]. Visible lack of electron-hole symmetry in these regions stems from explicit asymmetry of the bands and the choice of electron doping in the electrodes. With the growing amplitude and corresponding decrease of the conductance (Fig. 2) one sees

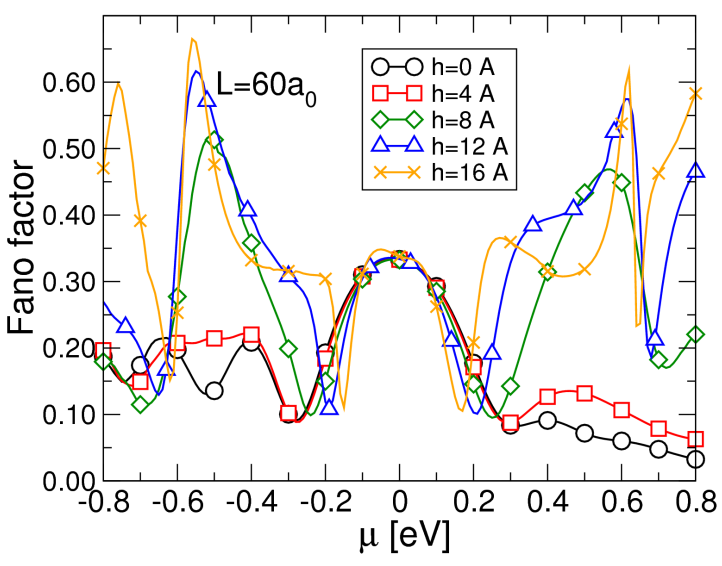

Fig. 3. Fano factors for transport through a single pe$\operatorname{riod}(L=60 \AA)$ of modulation as a function of the local chemical potential.

the general increase of $F$ with maximum values growing monotonically with $h_{0}$. This is in line with $F=1$ limit expected for uncorrelated tunneling regime.

\section{Summary}

In this paper the results of transport calculations for graphene with a simple sinusoidal modulation were presented. The dependence of the conductance through a single ripple on the chemical potential was found to be strongly non-monotonic with generally low values found for a strong modulation. Little difference was found between the model with and without hybridization which leads to the conclusion that it is of minor importance at least for the geometries studied.

\section{Acknowledgments}

This work was supported by the Polish Ministry of Science and Higher Education as a research project No. N N202 199239.

\section{References}

[1] K.S. Novoselov, A.K. Geim, S.V. Morozov, D. Jiang, Y. Zhang, S.V. Dubonos, I.V. Grigorieva, A.A. Firsov, Science 306, 666 (2004).

[2] K.S. Novoselov, A.K. Geim, S.V. Morozov, D. Jiang, M.I. Katsnelson, I.V. Grigorieva, S.V. Dubonos, A.A. Firsov, Nature 438, 197 (2005).

[3] A.H. Castro Neto, F. Guinea, N.M.R. Peres, K.S. Novoselov, A.K. Geim, Rev. Mod. Phys. 81, 109 (2009).

[4] J.C. Meyer, A.K. Geim, M.I. Katsnelson, K.S. Novoselov, T.J. Booth, S. Roth, Nature 446, 60 (2007).

[5] J.H.L.A. Fasolino, M.I. Katsnelson, Nature Mater. 6, 858 (2007).

[6] M. Ishigami, J.H. Chen, W.G. Cullen, M.S. Fuhrer, E.D. Williams, Nano Letters 7, 1643 (2007). 
[7] V. Geringer, M. Liebmann, T. Echtermeyer, S. Runte, M. Schmidt, R. Rückamp, M.C. Lemme, M. Morgenstern, Phys. Rev. Lett. 102, 076102 (2009).

[8] M. Gibertini, A. Tomadin, M. Polini, A. Fasolino, M.I. Katsnelson, Phys. Rev. B 81, 125437 (2010).

[9] F. Guinea, B. Horovitz, P. Le Doussal, Phys. Rev. B 77, 205421 (2008).

[10] F. Guinea, M.I. Katsnelson, M.A.H. Vozmediano, Phys. Rev. B 77, 075422 (2008).

[11] T.O. Wehling, A.V. Balatsky, A.M. Tsvelik, M.I. Katsnelson, A.I. Lichtenstein, Europhys. Lett. 84, 17003 (2008).

[12] S.V. Morozov, K.S. Novoselov, M.I. Katsnelson, F. Schedin, L.A. Ponomarenko, D. Jiang, A.K. Geim, Phys. Rev. Lett. 97, 016801 (2006).
[13] M. Katsnelson, A. Geim, Philos. Trans. R. Soc. A 366, 195 (2008).

[14] R. Saito, G. Dresselhaus, M.S. Dresselhaus, Physical Properties of Carbon Nanotubes, Imperial College Press, Londyn 2003.

[15] M. Zwierzycki, P.A. Khomyakov, A.A. Starikov, K. Xia, M. Talanana, P.X. Xu, V.M. Karpan, I. Marushchenko, I. Turek, G.E.W. Bauer, G. Brocks, P.J. Kelly, Phys. Status Solidi B 245, 623 (2008).

[16] M.I. Katsnelson, Eur. Phys. J. B 51, 157 (2006).

[17] J. Tworzydło, B. Trauzettel, M. Titov, A. Rycerz, C.W.J. Beenakker, Phys. Rev. Lett. 96, 246802 (2006). 